\title{
EIKONAL SOLUTION USING PHYSICS-INFORMED NEURAL NETWORKS
}

\author{
A PREPRINT
}

\author{
Umair bin Waheed \\ Department of Geosciences \\ King Fahd University of Petroleum and Minerals \\ Tariq Alkhalifah \\ Physical Sciences and Engineering Division \\ King Abdullah University of Science and Technology
}

\author{
Ehsan Haghighat \\ Department of Civil Engineering \\ Massachusetts Institute of Technology
}

\author{
Chao Song \\ Physical Sciences and Engineering Division \\ King Abdullah University of Science and Technology
}

\author{
Qi Hao \\ Center for Integrative Petroleum Research \\ King Fahd University of Petroleum and Minerals
}

July 17,2020

\begin{abstract}
The eikonal equation is utilized across a wide spectrum of science and engineering disciplines. In seismology, it regulates seismic wave traveltimes needed for applications like source localization, imaging, and inversion. Several numerical algorithms have been developed over the years to solve the eikonal equation. However, they suffer from computational bottleneck when repeated computations are needed for perturbations in the velocity model and/or the source location, particularly in large 3D models. Here, we employ the emerging paradigm of physics-informed neural networks (PINNs) to solve the eikonal equation. By minimizing a loss function formed by imposing the validity of the eikonal equation, we train a neural network to produce traveltimes that are consistent with the underlying partial differential equation. More specifically, to tackle point-source singularity, we use the factored eikonal equation. We observe sufficiently high traveltime accuracy for most applications of interest. We also demonstrate how machine learning techniques like transfer learning and surrogate modeling can be used to massively speed up traveltime computations for updated velocity models and source locations. These properties of the PINN eikonal solver are highly desirable in obtaining an efficient forward modeling engine for seismic inversion applications.
\end{abstract}

Keywords Eikonal equation · Physics-informed neural networks · Traveltimes · Forward modeling.

\section{Introduction}

The eikonal (from the Greek word $\epsilon \iota \kappa \omega \nu=$ image) equation is a first-order non-linear partial differential equation (PDE) encountered in the wave propagation and geometric optics literature. It was first derived by Sir William Rowan Hamilton in the year 1831 [1]. The eikonal equation finds its roots in both wave propagation theory and geometric optics. In wave propagation, the eikonal equation can be derived from the first term of the Wentzel-Kramers-Brillouin (WKB) expansion of the wave equation [2], whereas in geometric optics, it can be derived using Huygen's principle [3].

Despite its origins in optics, the eikonal equation finds applications in many science and engineering problems. To name a few, in image processing, it is used to compute distance fields from one or more points [4], inferring 3D surface shapes from intensity values in 2D images [5], image denoising [6], segmentation [7], and registration [8]. In robotics, the eikonal equation is extensively used for optimal path planning and navigation, e.g., for domestic robots [9], 
autonomous underwater vehicles [10], and Mars Rovers [11]. In computer graphics, the eikonal equation is used to compute geodesic distances for extracting shortest paths on discrete and parametric surfaces [12, 13]. In semi-conductor manufacturing, the eikonal equation is used for etching, deposition, and lithography simulations [14, 15]. Furthermore, and of primary interest to us, the eikonal equation is routinely employed in seismology to compute traveltime fields needed for many applications including statics and moveout correction [16], traveltime tomography [17], microseismic source localization [18], and Kirchhoff migration [19].

The fast marching method (FMM) and the fast sweeping method (FSM) are the two most commonly used algorithms for solving the eikonal equation. FMM belongs to the family of algorithms which are also referred to as single-pass methods. The first such algorithm is attributed to John Tsitsiklis [20], who used a control-theoretic discretization of the eikonal equation and emulated Dijkstra-like shortest path algorithm. However, a few months later, a finite-difference approach, also based on Dijkstra-like ordering and updating was developed [21]. The FMM combines entropy satisfying upwind schemes for gradient approximations and a fast sorting mechanism to solve the eikonal equation in a single-pass.

The FSM, on the other hand, is a multi-pass algorithm that combines Gauss-Seidel iterations with alternating sweeping ordering to solve the eikonal equation [22]. The idea behind the algorithm is that the characteristics of the eikonal equation can be divided into a finite number of pieces and information propagating along each piece can be accounted for by one of the sweeping directions. Therefore, FSM converges in a finite number of iterations, irrespective of the grid size.

Both FMM and FSM were initially proposed to solve the eikonal equation on rectangular grids. However, many different approaches have since been proposed, extending them to other discretizations and formulations. A detailed analysis and comparison of these fast methods can be found in [23].

On a different front, deep learning is fast emerging as a potential disruptive tool to tackle longstanding research problems across science and engineering disciplines [24]. Recent advances in the field of Scientific Machine Learning have demonstrated the largely untapped potential of deep learning for applications in scientific computing. The idea to use neural networks for solving PDEs has been around since the 1990s [25, 26]. However, recent advances in the theory of deep learning coupled with a massive increase in computational power and efficient graph-based implementation of new algorithms and automatic differentiation [27] have seen a resurgence of interest in using neural networks to approximate the solution of PDEs.

This resurgence is confirmed by the advances made in the recent literature on scientific computing. For example, [28] used a deep neural network (DNN) for modeling turbulence in fluid dynamics, while [29] proposed a deep learning algorithm to solve the nonlinear Black-Scholes equation, the Hamilton-Jacobi-Bellman equation, and the Allen-Cahn equation. Similarly, [30] developed a mesh-free algorithm based on deep learning for efficiently solving high-dimensional PDEs. In addition, [31] used a convolutional neural network to speed up the solution to a sparse linear system required to obtain a numerical solution of the Navier-Stokes equation.

Recently, Raissi et al. [32] developed a deep learning framework for the solution and discovery of PDEs. The so-called physics-informed neural network (PINN) leverages the capabilities of DNNs as universal function approximators. In contrast with the conventional deep learning approaches, PINNs restrict the space of admissible solutions by enforcing the validity of the underlying PDE governing the actual physics of the problem. This is achieved by using a simple feed-forward network leveraging automatic differentiation (AD), also known as algorithmic differentiation. PINNs have already demonstrated success in solving a wide range of non-linear PDEs, including Burgers, Schrödinger, Navier-Stokes, and Allen-Cahn equations.

In this paper, we develop a PINN-based algorithm for solving the eikonal equation. Based on a loss function defined by the underlying PDE, we train a DNN to yield the solution of the eikonal equation. To mitigate point-source singularity, we use the factored eikonal equation. Through tests on benchmark synthetic models, we study the accuracy properties of the proposed solver. We also explore how machine learning techniques like transfer learning and surrogate modeling can be used to massively speed up repeated traveltime computations with updated velocity models and/or source locations. These properties are highly desirable in reducing computational bottleneck for applications in seismic inversion.

The rest of the paper is organized as follows. We begin by describing the theoretical underpinnings of the algorithm. After presenting the eikonal equation and deep feed-forward neural networks, we briefly discuss important concepts including the approximation property of neural networks and automatic differentiation. Tying these concepts together, we then show how PINNs can be used to solve the eikonal equation. Next, we present numerical tests probing into the accuracy of the proposed framework on synthetic velocity models. We also explore the applicability of transfer learning and surrogate modeling to efficiently solve the eikonal equation. Next, we discuss the strengths and limitations of the approach including implications of this work on the field of numerical eikonal solvers. This is followed by some concluding remarks. 
A PREPRINT - JULY 17, 2020

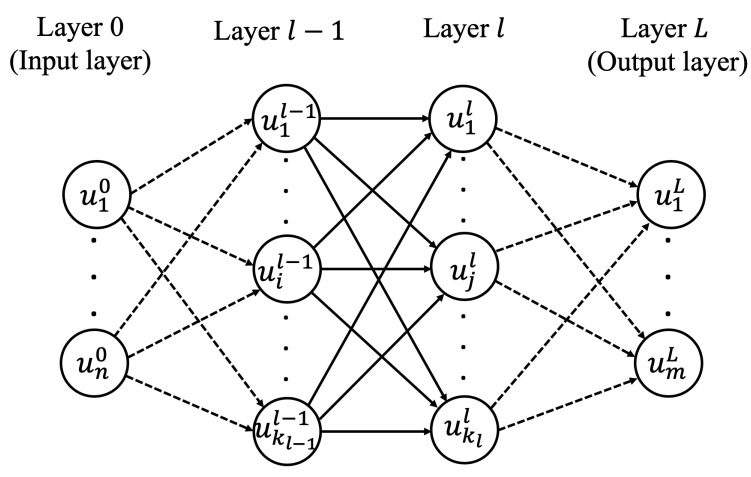

Figure 1: Schematic representation of a feed-forward neural network with $L-1$ hidden layers.

\section{Theory}

In this section, we first introduce the eikonal equation and the factorization idea. This is followed by a brief overview of deep neural networks and their capabilities as function approximators. Next, we briefly explain the concept of automatic differentiation. Finally, putting these pieces together, we present the proposed algorithm for solving the eikonal equation.

\subsection{Eikonal equation}

The eikonal equation is a non-linear, first-order, hyperbolic PDE of the form:

$$
\begin{aligned}
|\nabla T(\mathbf{x})|^{2} & =\frac{1}{v^{2}(\mathbf{x})}, \quad \forall \mathbf{x} \in \Omega, \\
T\left(\mathbf{x}_{\mathbf{s}}\right) & =0
\end{aligned}
$$

where $\Omega$ is a domain in $\mathbb{R}^{d}$ with $d$ as the space dimension, $T(\mathbf{x})$ is the traveltime or Euclidean distance to any point $\mathbf{x}$ from the point-source $\mathbf{x}_{\mathbf{s}}, v(\mathbf{x})$ is the velocity defined on $\Omega$, and $\nabla$ denotes the spatial differential operator. Equation (1) simply means the gradient of the arrival time surface is inversely proportional to the speed of the wavefront. This is also commonly known as the isotropic eikonal equation as the velocity is not a function of the wave propagation direction $(\nabla T /|\nabla T|)$.

To avoid the singularity due to the point-source [33], we consider the factored eikonal equation [34]. The factorization approach relies on factoring the unknown traveltime $(T(\mathbf{x}))$ into two functions. One of the functions is specified analytically, such that the other function is smooth in the source neighborhood. Specifically, we consider multiplicative factorization, i.e.,

$$
T(\mathbf{x})=T_{0}(\mathbf{x}) \tau(\mathbf{x})
$$

where $T_{0}(\mathbf{x})$ is known and $\tau(\mathbf{x})$ is the unknown function. Plugging equation 2 in equation 1 , we get the factored eikonal equation:

$$
\begin{gathered}
T_{0}^{2}|\nabla \tau|^{2}+\tau^{2}\left|\nabla T_{0}\right|^{2}+2 T_{0} \tau\left(\nabla T_{0} \cdot \nabla \tau\right)=\frac{1}{v^{2}(\mathbf{x})}, \\
\tau\left(\mathbf{x}_{\mathbf{s}}\right)=1 .
\end{gathered}
$$

The known factor $T_{0}$ is computed analytically using the expression:

$$
T_{0}(\mathbf{x})=\frac{\left|\mathbf{x}-\mathbf{x}_{\mathbf{s}}\right|}{v\left(\mathbf{x}_{\mathbf{s}}\right)},
$$

where $v\left(\mathbf{x}_{\mathbf{s}}\right)$ is the velocity at the source location.

\subsection{Deep feed-forward neural networks}

A feed-forward neural network is a set of neurons organized in layers in which evaluations are performed sequentially through the layers. It can be seen as a computational graph having an input layer, an output layer, and an arbitrary number of hidden layers. In a fully connected neural network, neurons in adjacent layers are connected with each other but neurons within a single layer share no connection. 
Thanks to the universal approximation theorem, a neural network with $n$ neurons in the input layer and $m$ neurons in the output layer can be used to represent a function $u: \mathbb{R}^{n} \rightarrow \mathbb{R}^{m}$ [35], as shown in Figure 1]. For illustration, we consider a network of $L+1$ layers starting with input layer 0 , the output layer $L$, and $L-1$ hidden layers. The number of neurons in each layer is denoted as $k_{0}=n, k_{1}, \cdots, k_{L}=m$. Each connection between the $i$-th neuron in layer $l-1$ and $j$-th neuron in layer $l$ has a weight $w_{j i}^{l}$ associated with it. Moreover, for each neuron in layer $l$, we have an associated bias term $b_{i}, i=1, \cdots, k_{l}$. Each neuron represents a mathematical operation, whereby it takes a weighted sum of its inputs plus a bias term and passes it through an activation function. The output from the $k$-th neuron in layer $l$ is given as [36]:

$$
u_{k}^{l}=\sigma\left(\sum_{j=1}^{k_{l}-1} w_{k j}^{l} u_{j}^{l-1}+b_{k}^{l}\right),
$$

where $\sigma()$ represents the activation function. Commonly used activation functions are the logistic sigmoid, the hyperbolic tangent, and the rectified linear unit [37]. By dropping the subscripts, we can write equation (5) compactly in the vectorial form:

$$
\mathbf{u}^{l}=\sigma\left(\mathbf{W}^{l} \mathbf{u}^{l-1}+\mathbf{b}^{l}\right)
$$

where $\mathbf{W}^{l}$ is the matrix of weights corresponding to connections between layers $l-1$ and $l, \mathbf{u}^{l}$ and $\mathbf{b}^{l}$ are vectors given by $u_{k}^{l}$ and $b_{k}^{l}$, respectively, and the activation function is applied element-wise. Computational frameworks, such as Tensorflow [38], can be used to efficiently evaluate data flow graphs like the one given in equation (6) efficiently using parallel execution. The input values can be defined as tensors (multi-dimensional arrays) and the computation of the outputs is vectorized and distributed across the available computational resources for efficient evaluation.

\subsection{Approximation property of neural networks}

Neural networks are well-known for their strong representational power. It has been shown that a neural network with a single hidden layer and a finite number of neurons can be used to represent any bounded continuous function to any desired accuracy. This is also known as the universal approximation theorem [39, 35]. It was later shown that by using a non-linear activation function and a deep network, the total number of neurons can be significantly reduced [40]. Therefore, we seek a trained deep neural network (DNN) that could represent the mapping between the input ( $x$ ) and the output $(\tau(\mathbf{x}))$ of the factored eikonal equation for a given velocity model $(v(\mathbf{x}))$.

It is worth noting that while neural networks are, in theory, capable of representing very complex functions compactly, finding the actual parameters (weights and biases) needed to solve a given PDE can be quite challenging.

\subsection{Automatic differentiation}

Solving a PDE using PINNs requires derivatives of the network's output with respect to the input. There are four possible ways to compute derivatives [27, 41]: (1) hand-coded analytical derivatives, (2) symbolic differentiation, (3) numerical approximation such as finite-difference, and (4) automatic differentiation (AD).

Manually working out the derivatives may be exact, but they are not automated, and thus, impractical. Symbolic differentiation is also exact, but it is memory intensive and prohibitively slow as one could end up with exponentially large expressions to evaluate. While numerical differentiation is easy to implement, it can be highly inaccurate due to round-off errors. On the contrary, AD uses exact expressions with floating-point values instead of symbolic strings and it involves no approximation error, resulting in accurate evaluation of derivatives at machine precision. However, an efficient implementation of AD can be non-trivial. Fortunately, many existing computational frameworks such as Tensorflow [38] and PyTorch [42] have made available efficiently implemented AD libraries. In fact, in deep learning, backpropagation [43], a generalized technique of $\mathrm{AD}$, has been the mainstay for training neural networks.

To understand how AD works, consider a simple fully-connected neural network with two inputs $\left(x_{1}, x_{2}\right)$, one output $(y)$, and one neuron in the hidden layer. Let us assume the network's weights and biases are assigned such that:

$$
\begin{aligned}
& \nu=2 x_{1}+3 x_{2}-1, \\
& h=\sigma(\nu)=\frac{1}{1+e^{-\nu}}, \\
& y=5 h+2,
\end{aligned}
$$

where $h$ represents the output from the neuron in the hidden layer computed by applying the sigmoid function $(\sigma)$ on the weighted sum of the inputs $(\nu)$.

To illustrate the idea, let us say we are interested in computing partial derivatives $\frac{\partial y}{x_{1}}$ and $\frac{\partial y}{x_{2}}$ at $\left(x_{1}, x_{2}\right)=(1,-1)$. AD requires one forward pass and backward pass through the network to compute these derivatives as detailed in Table 1 
To compute high-order derivatives, AD can be applied recursively through the network in the same manner. For a deeper understanding of $\mathrm{AD}$, we refer the interested reader to [44].

\begin{tabular}{|c|c|}
\hline Forward pass & Reverse pass \\
\hline $\begin{array}{l}x_{1}=1 \\
x_{2}=-1\end{array}$ & $\frac{\partial y}{\partial y}=1$ \\
\hline $\begin{array}{l}\nu=2 x_{1}+3 x_{2}-1=-2 \\
h=\frac{1}{1+e^{-\nu}}=0.119\end{array}$ & $\begin{aligned} \frac{\partial y}{\partial h} & =\frac{\partial(5 h+2)}{\partial h}=5 \\
\frac{\partial y}{\partial \nu} & =\frac{\partial y}{\partial h} \cdot \frac{\partial h}{\partial \nu}=5 \times \frac{e^{-\nu}}{\left(1+e^{-\nu}\right)^{2}} \\
& =0.525\end{aligned}$ \\
\hline$y=5 h+2=2.596$ & $\begin{array}{l}\frac{\partial y}{\partial x_{1}}=\frac{\partial y}{\partial \nu} \cdot \frac{\partial \nu}{\partial x_{1}}=\frac{\partial y}{\partial \nu} \times 2=1.050 \\
\frac{\partial y}{\partial x_{2}}=\frac{\partial y}{\partial \nu} \cdot \frac{\partial \nu}{\partial x_{2}}=\frac{\partial y}{\partial \nu} \times 3=1.575\end{array}$ \\
\hline
\end{tabular}

Table 1: Example of forward and reverse pass computations needed by AD to compute partial derivates of the output with respect to the inputs at $\left(x_{1}, x_{2}\right)=(1,-1)$ for the expressions given in equation (7).

\subsection{Solving the eikonal equation}

To solve the eikonal equation (1), we leverage the capabilities of neural networks as function approximators and define a loss function that minimizes the residual of the factored eikonal equation at a chosen set of training (collocation) points. This is achieved with (i) a DNN approximation of the unknown traveltime field variable $\tau(\mathbf{x})$; (ii) a loss function incorporating the eikonal equation and sampled on a collocation grid; (iii) a differentiation algorithm, i.e. AD in this case, to evaluate partial derivatives of $\tau(\mathbf{x})$ with respect to the spatial coordinates.

To illustrate the idea, let us consider a two-dimensional domain $\Omega \in \mathbb{R}^{2}$ where $\mathbf{x}=(x, z) \in[0,2]$, as shown in Figure 2 A source term is considered at $\mathbf{x}_{\mathbf{s}}=\left(x_{s}, z_{s}\right)$, where $\tau\left(\mathbf{x}_{\mathbf{s}}\right)=1$. The unknown traveltime factor $\tau(\mathbf{x})$ is approximated by a multilayer DNN $\mathcal{N}_{\tau}$, i.e. $\tau(\mathbf{x}) \approx \hat{\tau}(\mathbf{x})=\mathcal{N}_{\tau}(\mathbf{x} ; \boldsymbol{\theta})$, where $\mathbf{x}=(x, z)$ are network inputs, $\hat{\tau}$ is the network output, and $\boldsymbol{\theta}$ represents the set of all trainable parameters of the network.

The loss function can now be constructed using a mean-squared-error (MSE) norm as:

$$
\mathfrak{J}=\frac{1}{N_{I}} \sum_{\mathbf{x}^{*} \in I}\|\mathcal{L}\|^{2}+\frac{1}{N_{I}} \sum_{\mathbf{x}^{*} \in I}\|\mathcal{H}(-\hat{\tau}) \mid \hat{\tau}\|^{2}+\left\|\hat{\tau}\left(\mathbf{x}_{\mathbf{s}}\right)-1\right\|^{2},
$$

where

$$
\mathcal{L}=T_{0}^{2}|\nabla \hat{\tau}|^{2}+\hat{\tau}^{2}\left|\nabla T_{0}\right|^{2}+2 T_{0} \hat{\tau}\left(\nabla T_{0} . \nabla \hat{\tau}\right)-\frac{1}{v^{2}(\mathbf{x})},
$$

forms the residual of the factored eikonal equation.

The first term on the right side of equation 8 imposes the validity of the factored eikonal equation on a given set of training points $\mathbf{x}^{*} \in I$, with $N_{I}$ as the number of sampling points. The second term forces the solution $\hat{\tau}$ to be positive by penalizing negative solutions using the Heaviside function $\mathcal{H}()$. The last term requires the solution to be unity at the point-source $\mathbf{x}_{\mathbf{s}}=\left(x_{s}, z_{s}\right)$.

Network parameters $\boldsymbol{\theta}$ are then identified by minimizing the loss function (8) on a set of sampling (training) points $\mathbf{x}^{*} \in I$, i.e.,

$$
\arg \min _{\boldsymbol{\theta}} \mathfrak{J}\left(\mathbf{x}^{*} ; \boldsymbol{\theta}\right)
$$

Once the DNN is trained, we evaluate the network on a set of regular grid-points in the computational domain to obtain the unknown traveltime part. The final traveltime solution is obtained by multiplying it with the known traveltime part, i.e.,

$$
\hat{T}(\mathbf{x})=T_{0}(\mathbf{x}) \hat{\tau}(\mathbf{x})
$$




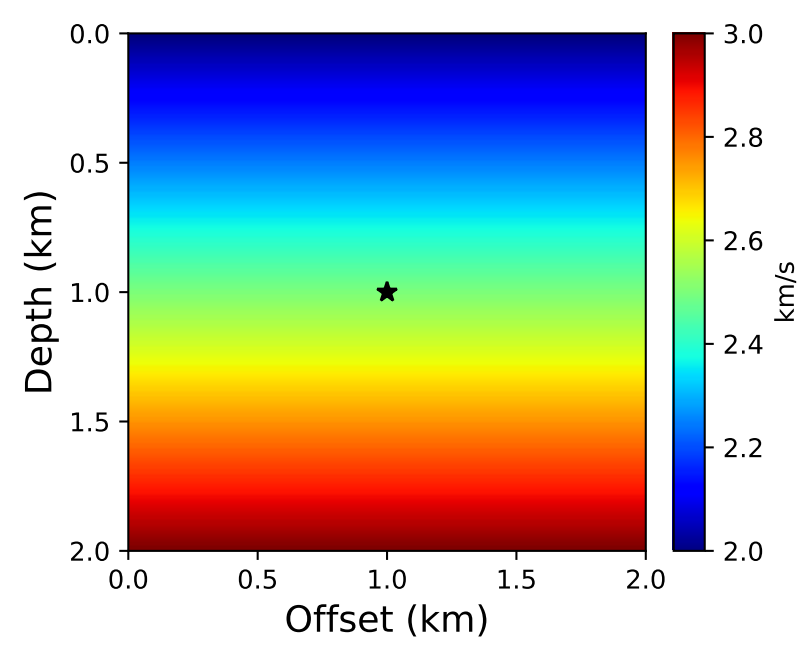

Figure 2: A velocity model with a constant velocity gradient of $0.5 \mathrm{~s}^{-1}$ in the vertical direction. The velocity at zero depth is equal to $2 \mathrm{~km} / \mathrm{s}$ and it increases linearly to $3 \mathrm{~km} / \mathrm{s}$ at a depth of $2 \mathrm{~km}$. Black star indicates the point-source location used for the tests.

It is worth emphasizing that the proposed approach is different from traditional (or non-physics constrained) deep learning techniques. The training of the network here refers to the tuning of weights and biases of the network such that the resulting solution minimizes the loss function $\mathfrak{J}$ on a given set of training points. Also noteworthy is the fact that, contrary to supervised learning applications, the network here learns without any labeled set. To understand this point, consider a randomly initialized network, which will output a certain value $\hat{\tau}_{i, j}$ for each point $(i, j)$ in the training set. These output values will be used to calculate the residual using equation 8 Based on this residual, the network adjusts its weights and biases allowing it to produce $\hat{\tau}$ that adheres to the underlying factored eikonal equation (3).

\section{Numerical Tests}

In this section, we test the proposed PINN eikonal solver for computing traveltimes emanating from a point-source. We consider models with smoothly varying velocity fields along the horizontal and vertical directions, and a highly heterogeneous portion from the well-known Marmousi model. For each case presented below, we minimize the loss function given in equation 8 using the Adam optimizer with full-batch optimization. Moreover, the activation function used for the hidden layers of the network is the inverse tangent (arctan) whereas the final layer is linear. The PINN framework is implemented using the SciANN package [45] - a high level Tensorflow wrapper for scientific computations. For comparison, we use the first-order finite-difference fast marching solution, which is routinely used for traveltime computations in seismological applications.

First, we consider a $2 \times 2 \mathrm{~km}^{2}$ model with vertically varying velocity. The velocity at zero depth is $2 \mathrm{~km} / \mathrm{s}$ and it increases linearly with a gradient of $0.5 \mathrm{~s}^{-1}$. We consider the point-source to be located at $(1 \mathrm{~km}, 1 \mathrm{~km})$. The model is shown in Figure 2 with the black star depicting the point-source location. For a model with a constant velocity gradient, the analytical traveltime solution is given as [46]:

$$
T(\mathbf{x})=\frac{1}{\sqrt{g_{v}^{2}+g_{h}^{2}}} \operatorname{arccosh}\left(1+\frac{\left(g_{v}^{2}+g_{h}^{2}\right)\left|\mathbf{x}-\mathbf{x}_{\mathbf{s}}\right|^{2}}{2 v(\mathbf{x}) v\left(\mathbf{x}_{\mathbf{s}}\right)}\right),
$$

where $T(\mathbf{x})$ is the traveltime value at some grid point $\mathbf{x}=(x, y)$, from a point-source located at $\mathbf{x}_{\mathbf{s}}$. Likewise, $v(\mathbf{x})$ is the velocity at the grid-point $\mathbf{x}$ and $v\left(\mathbf{x}_{\mathbf{S}}\right)$ is the velocity at the point-source location. The velocity gradients along the vertical and horizontal dimensions are denoted by $g_{v}$ and $g_{h}$, respectively. Therefore, for the model in Figure 2 , $g_{v}=0.5 \mathrm{~s}^{-1}, g_{h}=0 \mathrm{~s}^{-1}, \mathbf{x}_{\mathbf{s}}=(1 \mathrm{~km}, 1 \mathrm{~km})$, and $v\left(\mathbf{x}_{\mathbf{s}}\right)=2.5 \mathrm{~km} / \mathrm{s}$.

We begin the tests by studying the predictive accuracy of the PINN eikonal solver for different network architectures. For the considered point-source, we compute traveltime solutions by using a number of different architectures formed by varying the number of hidden layers and the number of neurons in each hidden layer. For each architecture, we use 2600 training points within the computational domain. As shown in Table 2 , we compute relative $\mathcal{L}_{2}$ error between the predicted solution and the analytical solution computed using equation 12. As expected, we observe that as we 


\begin{tabular}{|c||c|c|c|c|c|}
\hline Layers & 2 & 4 & 6 & 8 & 10 \\
\hline \hline 2 & $9.59 \mathrm{e}-03$ & $2.12 \mathrm{e}-03$ & $1.28 \mathrm{e}-03$ & $7.91 \mathrm{e}-04$ & $5.25 \mathrm{e}-04$ \\
\hline 4 & $1.96 \mathrm{e}-03$ & $1.45 \mathrm{e}-03$ & $5.06 \mathrm{e}-04$ & $3.47 \mathrm{e}-04$ & $4.04 \mathrm{e}-04$ \\
\hline 6 & $2.96 \mathrm{e}-03$ & $8.31 \mathrm{e}-04$ & $9.34 \mathrm{e}-04$ & $5.05 \mathrm{e}-04$ & $2.58 \mathrm{e}-04$ \\
\hline 8 & $7.78 \mathrm{e}-04$ & $4.45 \mathrm{e}-04$ & $1.01 \mathrm{e}-03$ & $8.16 \mathrm{e}-04$ & $5.95 \mathrm{e}-04$ \\
\hline 10 & $1.99 \mathrm{e}-03$ & $3.10 \mathrm{e}-04$ & $3.99 \mathrm{e}-04$ & $4.17 \mathrm{e}-04$ & $3.16 \mathrm{e}-04$ \\
\hline
\end{tabular}

Table 2: Relative $\mathcal{L}_{2}$ errors between the predicted and the analytical solutions for different number of hidden layers and neurons per layer. The velocity model used is shown in Figure 2 and the point-source is located at $(1 \mathrm{~km}, 1 \mathrm{~km})$.

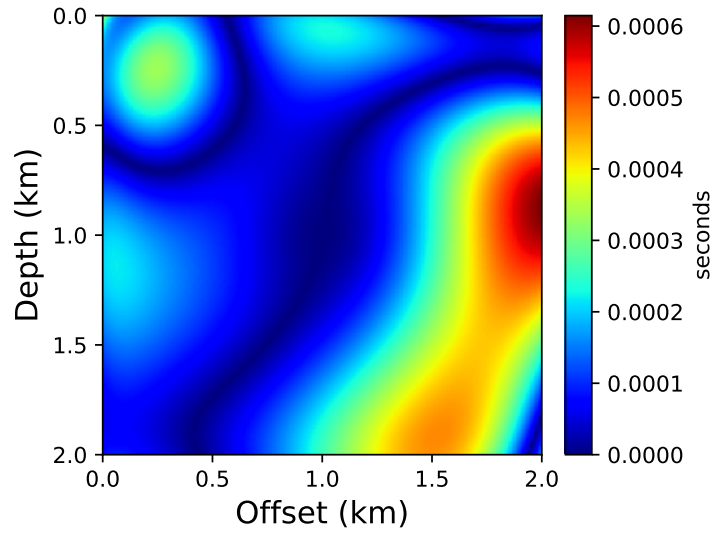

(a)

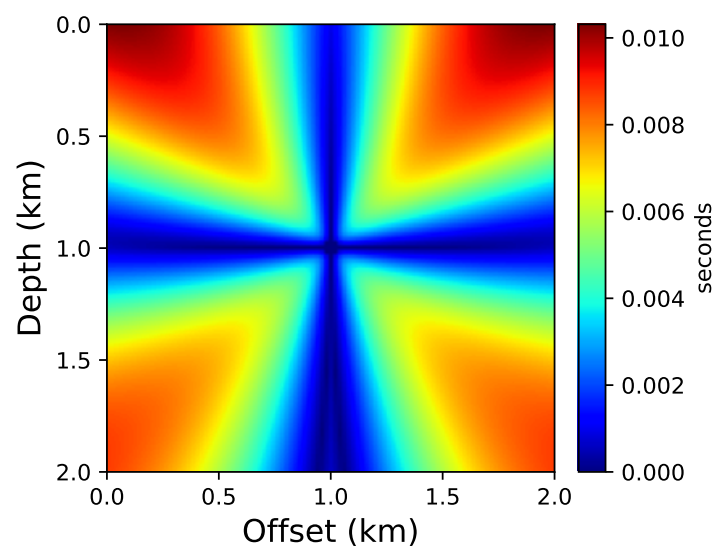

(b)

Figure 3: The absolute traveltime errors for the PINN eikonal solution (a) and the first-order fast marching solution (b) for the velocity model and the source location shown in Figure 2

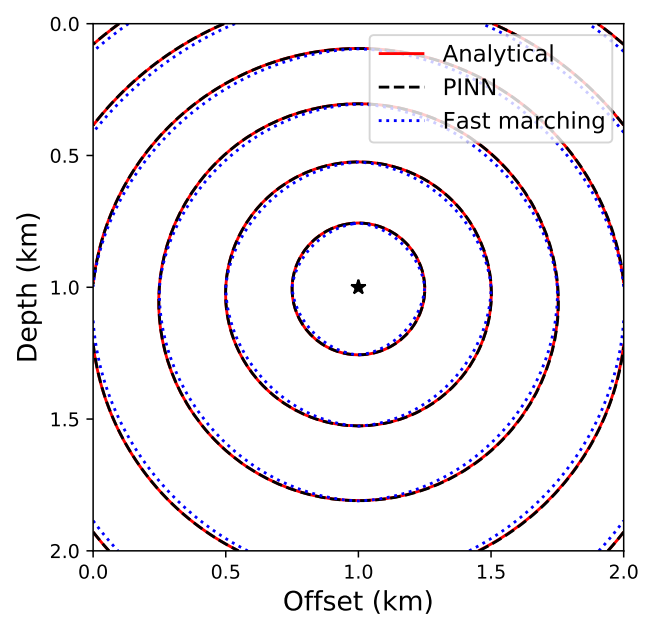

Figure 4: Traveltime contours for the analytical solution (red), PINN eikonal solution (dashed black), and the first-order fast marching solution (dotted blue). The velocity model and the source location considered are shown in Figure 2

increase the number of layers and neurons, the predictive accuracy of the network is improved as it can approximate more complex functions.

Next, in Figure 3(a) we show the absolute traveltime errors for PINN eikonal solver considering the same velocity model and source position. The network contains 10 hidden layers and 20 neurons in each layer and is trained on 2000 randomly selected points in the computational domain. Once the network is trained, we evaluate the network on a 
regular grid with $20 \mathrm{~m}$ grid spacing along the horizontal and vertical dimensions. For comparison, we also plot absolute traveltime errors for the first-order fast marching solution in Figure 3(b) on the same regular grid. We observe that despite using only 2000 randomly selected grid points, the PINN eikonal solution is significantly more accurate than the first-order fast marching solution. As can be seen in Figure 3(b), the fast marching solution suffers from large errors in the diagonal direction, whereas the errors for the PINN eikonal solver are more randomly distributed. We also plot traveltime contours in Figure 4 comparing the analytical solution with the PINN eikonal solution and the first-order fast marching solution visually.

Next, we investigate the applicability of transfer learning to the PINN eikonal solver. Transfer learning is a machine learning technique that relies on storing knowledge gained while solving one problem and applying it to a different but related problem. We explore if the network trained for the previous example can be used to compute the solution for a different source location and velocity model. To this end, we consider a different velocity model with a vertical gradient of $1 \mathrm{~s}^{-1}$ and a horizontal gradient of $0.5 \mathrm{~s}^{-1}$. The point-source is also relocated to $(1.4 \mathrm{~km}, 0.3 \mathrm{~km})$ as shown in Figure 5 .

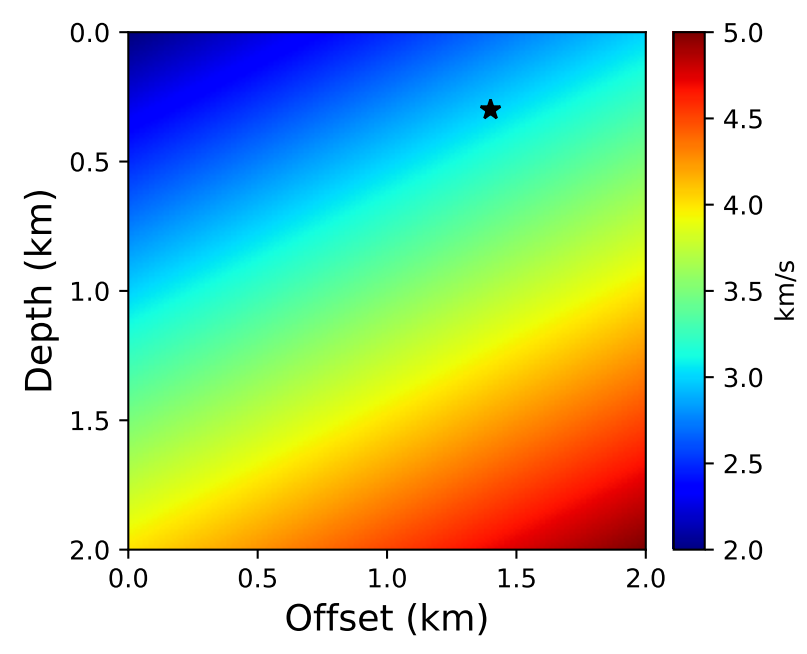

Figure 5: A velocity model with a constant vertical velocity gradient of $1 \mathrm{~s}^{-1}$ and a horizontal velocity gradient of $0.5 \mathrm{~s}^{-1}$. Black star indicates the point-source location used for the test.

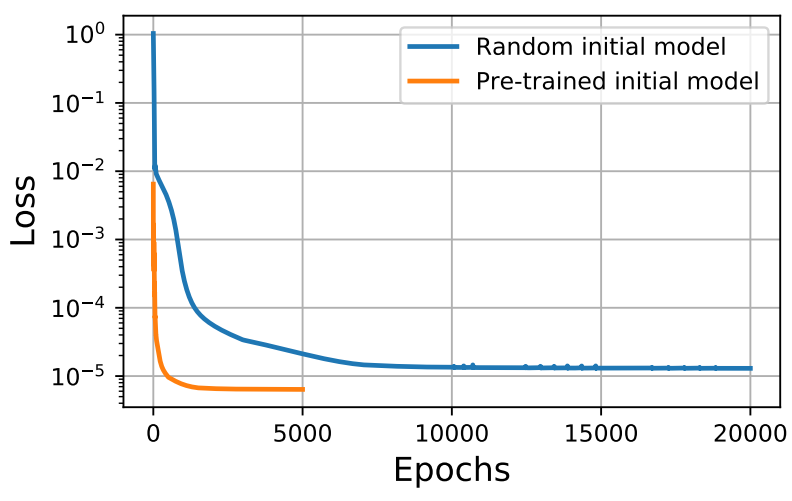

Figure 6: A comparison of convergence history for training of the examples shown in Figure 2 (blue) and Figure 5 (orange). The former example used a network with random initialization, whereas the network of the latter example was initialized using pre-trained weights from the former example.

To train the network for this case, instead of initializing the network with random weights, we use the weights from the network trained for the previous example. We observe that the network converges much faster than the previous example, which was randomly initialized, as shown in Figure 6 This is a highly desirable property of the PINN eikonal solver since seismic applications, such as earthquake source localization or seismic imaging, require repeated traveltime 


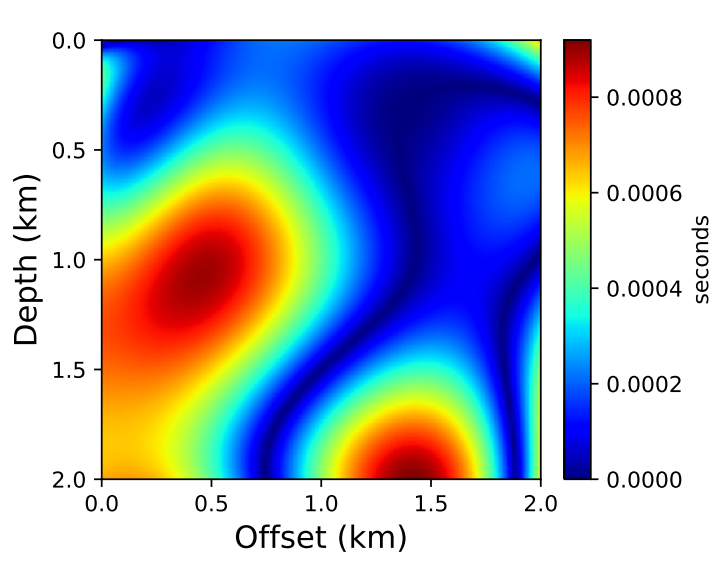

(a)

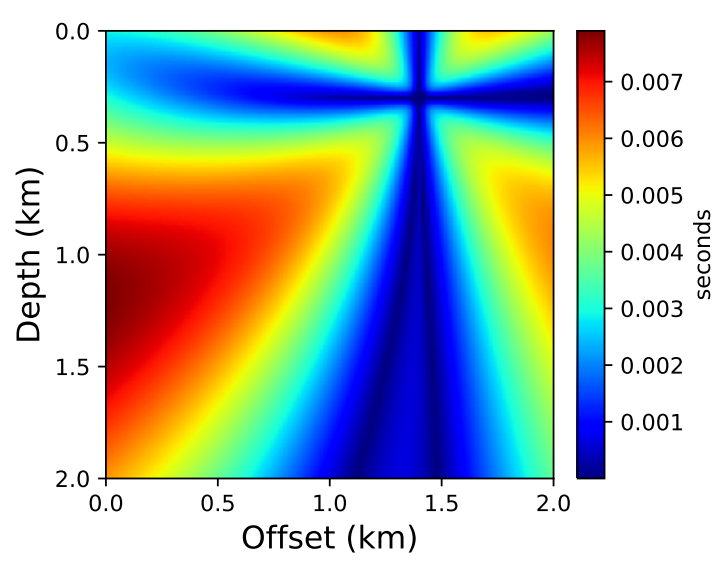

(b)

Figure 7: The absolute traveltime errors for the PINN eikonal solution (a) and the first-order fast marching solution (b) for the velocity model and the source location shown in Figure 5 .

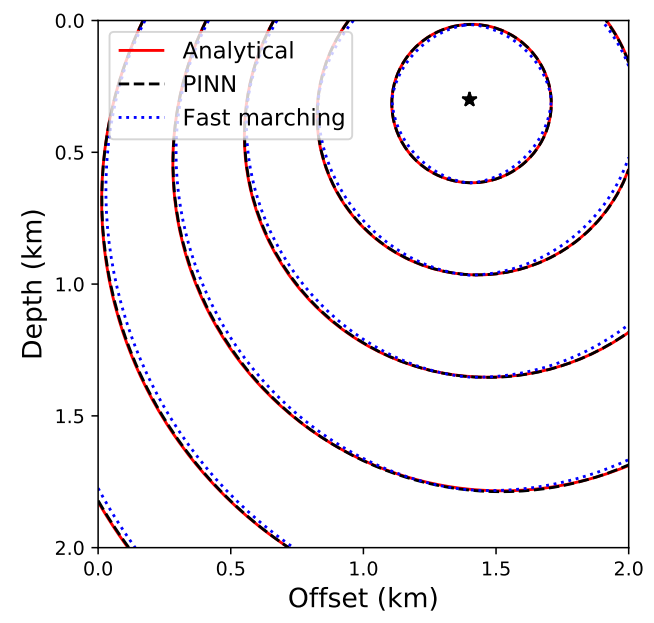

Figure 8: Traveltime contours for the analytical solution (red), PINN eikonal solution (dashed black), and the first-order fast marching solution (dotted blue). The velocity model and the source location considered are shown in Figure 5 .

computations for multiple source locations and updated velocity models. In comparison, conventional numerical algorithms, such as the fast marching method, require the same computational effort for even a slight variation in the velocity model or source location, which is a major source of the computational bottleneck, particularly in large 3D models.

Figure 7 compares the absolute traveltime errors computed using the PINN eikonal solution with the pre-trained initial model and the first-order fast marching solution. We observe that despite using significantly fewer epochs than in the previous example, the solution accuracy is not compromised. The traveltime contours, shown in Figure 8 , confirm this observation visually.

Next, we explore if a PINN model trained on solutions computed for various source locations in a given velocity model can be used as a surrogate model. To do this, the network is modified to include the source location $\mathbf{x}_{\mathbf{s}}=\left(x_{s}, z_{s}\right)$ as inputs in addition to the grid points $\mathbf{x}=(x, z)$. We train the network on solutions computed for 16 sources located at regular intervals in a constant vertical gradient model, as shown in Figure 9. Through this training process, the network learns the mapping between source locations, $\mathbf{x}_{\mathbf{s}}$, and the corresponding traveltime fields, $T(\mathbf{x})$. Once the surrogate model is trained with source locations as additional input parameters, traveltime fields for new source locations for the 
A PREPRINT - JULY 17, 2020

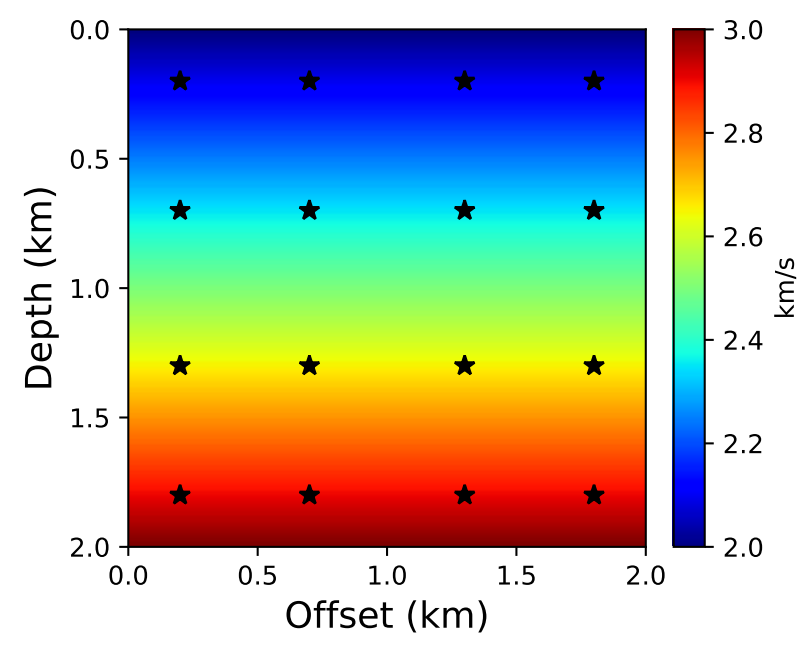

Figure 9: A velocity model with a constant velocity gradient of $0.5 \mathrm{~s}^{-1}$ in the vertical direction. Black stars indicate locations of sources used to train the network as a surrogate model.

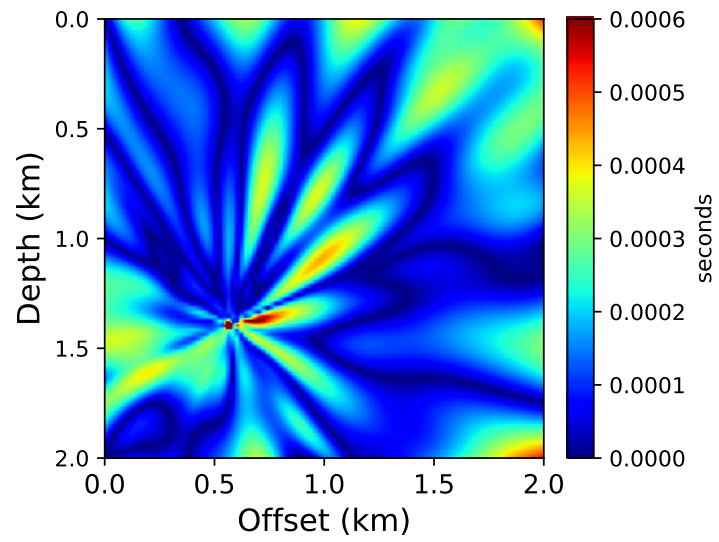

(a)

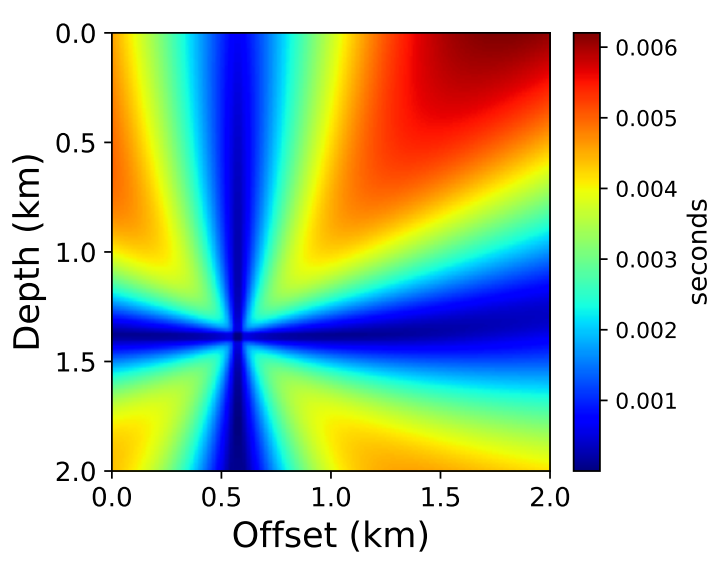

(b)

Figure 10: The absolute traveltime errors for the solution computed using the PINN surrogate model (a) and the first-order fast marching solution (b) for the velocity model shown in Figure 9 and a randomly chosen source point.

given velocity model can be computed rapidly with just a single evaluation of the network. This is similar to obtaining an analytic solver as no training is needed for computing traveltimes corresponding to additional source locations.

To demonstrate if the surrogate model approach yields an accurate solution, we use the trained surrogate model to compute traveltime solutions for a randomly chosen source location in the model. We can confirm by looking at the absolute traveltime errors, shown in Figure 10, that the trained surrogate model yields a highly accurate solution compared to the first-order fast marching solution even though no training is performed for this randomly chosen source point. This observation is confirmed by seeing the traveltime contours in Figure 8

Moreover, transfer learning can be used to efficiently build surrogate models for updated velocity models, i.e. by initializing the PINN surrogate model for the updated velocities using weights from the already trained surrogate model. Therefore, the transfer learning technique combined with surrogate modeling can be used to build a highly efficient traveltime modeling algorithm for seismic inversion compared to conventional numerical algorithms that do not afford such flexibility.

Finally, we test the PINN eikonal solver on a highly heterogeneous portion of the Marmousi model as shown in Figure 12 We consider a source located at $(1 \mathrm{~km}, 1 \mathrm{~km})$. This is a particularly challenging model due to sharp velocity 
A PREPRINT - JULY 17, 2020

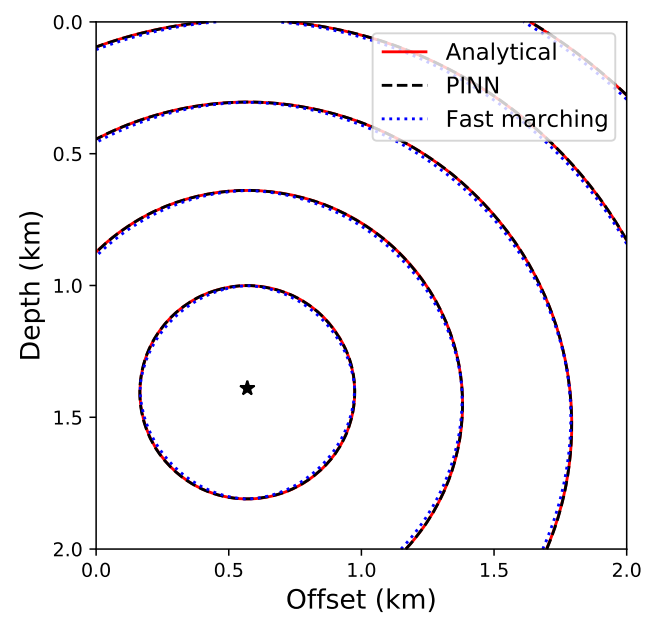

Figure 11: Traveltime contours for solutions obtained using the analytical formula (red), the PINN surrogate model (dashed black), and the first-order fast marching solver (dotted blue). The velocity model considered is shown in Figure 9 .

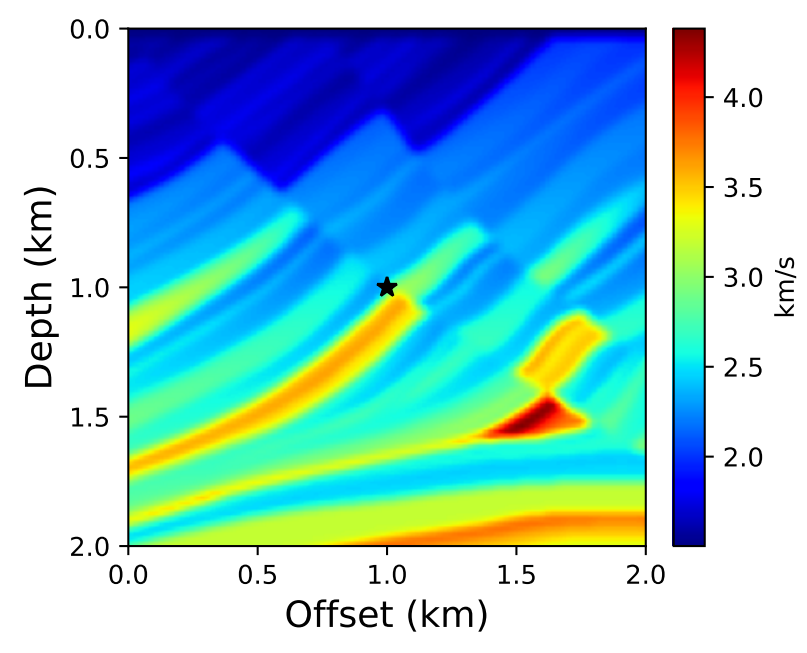

Figure 12: A highly heterogeneous portion of the Marmousi model used for traveltime computation. Black star indicates the point-source location used for the test.

variations. We train the network on 5000 randomly selected points in the computational domain. Once the network is trained, we evaluate the network on a regular grid with $20 \mathrm{~m}$ grid spacing along the horizontal and vertical dimensions. For comparison, we also compute the traveltime solution using the first-order fast marching method on the same regular grid. The absolute traveltime errors for both the approaches are compared in Figure 13 and the traveltime contours are shown in Figure 14. We observe significantly better accuracy for the PINN eikonal solver compared to the first-order fast marching method. However, for the PINN eikonal solution, we observe a small region of large errors indicated by the red arrow in Figure 14. This coincides with the region of the largest velocity gradient indicating that PINN models may suffer from inaccuracies in such cases. Compared to previous examples, we also observe slower convergence for the Marmousi example, which is explained by the highly non-convex loss function in this case.

\section{Discussion}

In a conventional deep learning application, a neural network is trained by minimizing a loss function that typically measures the mismatch between the network's predicted output and some known outputs, also known as training data. However, there are several limitations associated with such models that solely rely on a labeled dataset and are oblivious 


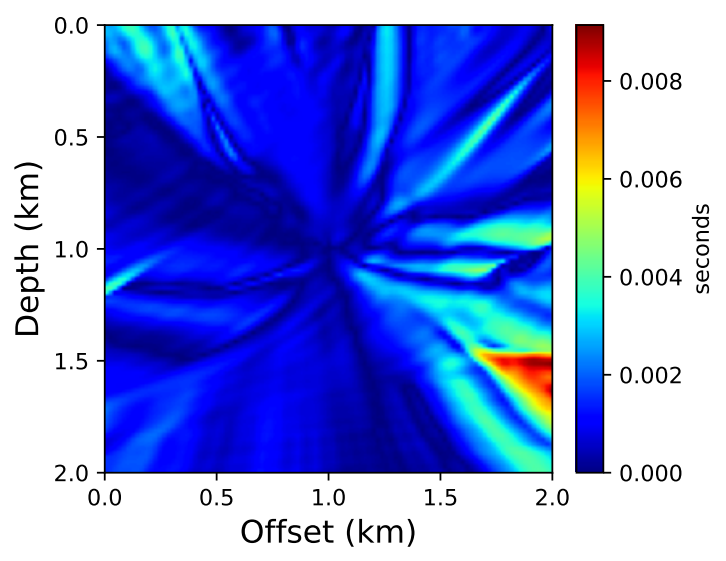

(a)

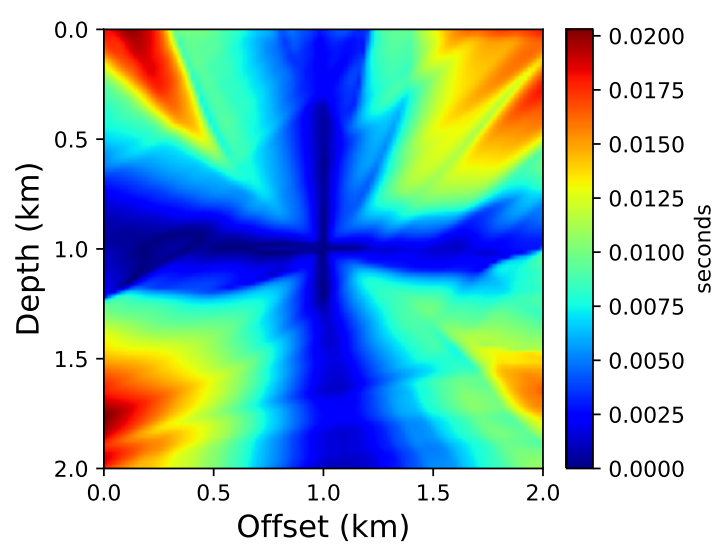

(b)

Figure 13: The absolute traveltime errors for the PINN eikonal solution (a) and the first-order fast marching solution (b) for the velocity model and the source location shown in Figure 12

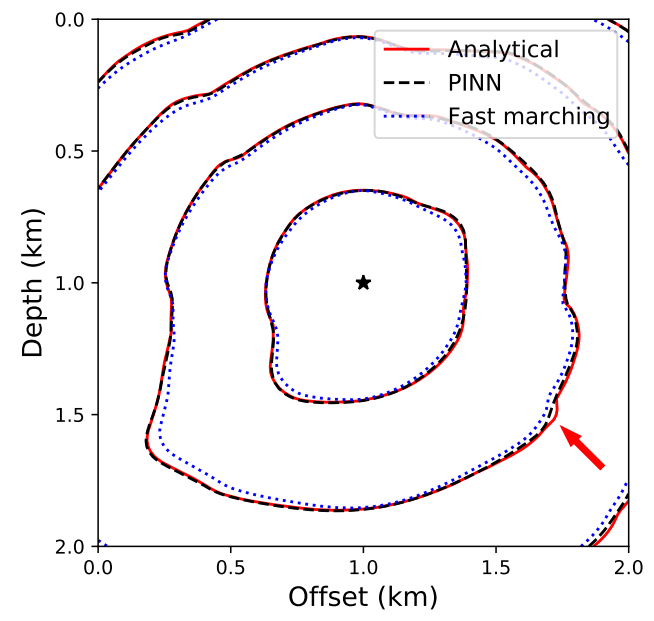

Figure 14: Traveltime contours for the reference solution (red), PINN eikonal solution (dashed black), and the first-order fast marching solution (dotted blue). The velocity model and the source location considered are shown in Figure 12 . The red arrow indicates visible region of inaccuracy in the PINN eikonal solution.

to the scientific principles governing real-world phenomena. For cases when the available training and test data are insufficient, such models often learn spurious relationships that are misleading. However, the biggest concern of such a data-driven model is the lack of scientific consistency of their predictions to known physical laws that have been the cornerstone of knowledge discovery across scientific disciplines for centuries.

Therefore, in the present work, we propose an eikonal solver based on the framework of physics-informed neural networks [32]. We leverage the capabilities of neural networks as universal function approximators [35] and define a loss function to minimize the residual of the governing eikonal equation at a chosen set of training points. This is achieved with a simple feed-forward neural network leveraging the concept of automatic differentiation [27]. Through numerical tests, we observe that the proposed algorithm yield sufficiently accurate traveltimes for most seismic applications of interest. We demonstrate this by comparing the accuracy of the proposed approach against the first-order fast marching solution, which is a popular numerical algorithm for solving the eikonal equation.

We find that the training cost of the PINN eikonal solver can be reduced by using a few randomly selected grid points from the computational domain, particularly for smooth velocity models. For tests on the smoothly varying velocity models with constant horizontal and vertical gradients, we randomly selected only $20 \%$ of the total grid points, whereas we used $50 \%$ of the total grid points for the Marmousi model example. We also observe that the transfer learning 
technique can be used to speed up the convergence of the network for new source locations and/or updated velocity models by initializing the PINN model with the weights of a previously trained network. Moreover, having computed solutions corresponding to a few sources for a given velocity model, we can also build a surrogate model with respect to the source locations by adding them as input parameters. This essentially means that this surrogate model can then be used to compute traveltime fields corresponding to new source locations for the same velocity model just by a single evaluation of the network. These observations effectively demonstrate the potential of the proposed approach in massively speeding up many seismic applications that rely on repeated traveltime computations for multiple source locations and velocity models. Moreover, the extension of the proposed framework to more complex eikonal equations, for example the anisotropic eikonal equation, is much simpler compared to the conventional numerical algorithms.

Nevertheless, there are a few challenges in our observation that need further investigation. Most importantly, we observe slow convergence for velocity models with sharp variations. This needs to be addressed particularly for areas with complex subsurface geologic structures. Possible solutions to this problem may include a denser sampling of training points around parts of the velocity model with a large velocity gradient [47] or the use of non-local PINN framework [48]. Another challenge is concerning the optimal choice of the network hyper-parameters that are often highly problem dependent. Recent advances in the field of meta-learning may enable automated selection of optimum architectures.

\section{Conclusions}

We proposed a method to solve the eikonal equation using the deep learning framework. Through tests on benchmark synthetic models, we show that the accuracy of the proposed approach is better than the first-order fast marching solution. Depending on the heterogeneity in the velocity model, we also note that training is needed for only a fraction of the total grid points in the computational domain to reliably reconstruct the solution. We also observed that transfer learning can be used to speed up convergence for new velocity models and/or source locations. Moreover, having computed solutions corresponding to a few source locations for a given velocity model, surrogate modeling can be used to train a network to instantly yield traveltime solutions corresponding to new source locations. These properties, not afforded by the conventional numerical algorithms, potentially allow us to massively speed up seismic inversion applications, particularly for large 3D models. We demonstrated the proposed framework on 2D models for the simplicity of illustration. The extension to $3 \mathrm{D}$ velocity models is straightforward.

\section{References}

[1] Jaume Masoliver and Ana Ros. From classical to quantum mechanics through optics. European journal of physics, 31(1):171, 2009.

[2] Demetrius T Paris and Frank Kenneth Hurd. Basic electromagnetic theory. McGraw-Hill Education, 1969.

[3] Vladimir Igorevich Arnold. Mathematical methods of classical mechanics, volume 60. Springer Science \& Business Media, 2013.

[4] David Adalsteinsson and James A Sethian. A fast level set method for propagating interfaces. J. Comput. Phys, 118(2), 1994.

[5] Elisabeth Rouy and Agnès Tourin. A viscosity solutions approach to shape-from-shading. SIAM Journal on Numerical Analysis, 29(3):867-884, 1992.

[6] Ravikanth Malladi and James A Sethian. A unified approach to noise removal, image enhancement, and shape recovery. IEEE Transactions on Image Processing, 5(11):1554-1568, 1996.

[7] Christopher Alvino, Gozde Unal, Greg Slabaugh, Bertrand Peny, and Tong Fang. Efficient segmentation based on eikonal and diffusion equations. International Journal of Computer Mathematics, 84(9):1309-1324, 2007.

[8] Zhujiang Cao, Shiyan Pan, Rui Li, Ramya Balachandran, J Michael Fitzpatrick, William C Chapman, and Benoit M Dawant. Registration of medical images using an interpolated closest point transform: method and validation. Medical image analysis, 8(4):421-427, 2004.

[9] Rodrigo Ventura and Aamir Ahmad. Towards optimal robot navigation in domestic spaces. In Robot Soccer World Cup, pages 318-331. Springer, 2014.

[10] Clment Petres, Yan Pailhas, Pedro Patron, Yvan Petillot, Jonathan Evans, and David Lane. Path planning for autonomous underwater vehicles. IEEE Transactions on Robotics, 23(2):331-341, 2007.

[11] Santiago Garrido, David Álvarez, and Luis Moreno. Path planning for mars rovers using the fast marching method. In Robot 2015: Second Iberian Robotics Conference, pages 93-105. Springer, 2016. 
[12] Ron Kimmel and James A Sethian. Computing geodesic paths on manifolds. Proceedings of the national academy of Sciences, 95(15):8431-8435, 1998.

[13] Alon Spira and Ron Kimmel. An efficient solution to the eikonal equation on parametric manifolds. Interfaces and Free Boundaries, 6(3):315-327, 2004.

[14] John Joseph Helmsen, Elbridge Gerry Puckett, Phillip Colella, and Milo Dorr. Two new methods for simulating photolithography development in 3d. In Optical Microlithography IX, volume 2726, pages 253-261. International Society for Optics and Photonics, 1996.

[15] D Adalsteinsson and JA Sethian. Level set methods for etching, deposition and photolithography development. Journal of Technology Computer Aided Design TCAD, pages 1-67, 1996.

[16] Don C Lawton. Computation of refraction static corrections using first-break traveltime differences. Geophysics, 54(10):1289-1296, 1989.

[17] Cédric Taillandier, Mark Noble, Hervé Chauris, and Henri Calandra. First-arrival traveltime tomography based on the adjoint-state method. Geophysics, 74(6):WCB1-WCB10, 2009.

[18] Vladimir Grechka, Alejandro De La Pena, Estelle Schisselé-Rebel, Emmanuel Auger, and Pierre-Francois Roux. Relative location of microseismicity. Geophysics, 80(6):WC1-WC9, 2015.

[19] Gilles Lambare, Stephane Operto, Pascal Podvin, and Philippe Thierry. 3d ray+ born migration/inversion—part 1: Theory. Geophysics, 68(4):1348-1356, 2003.

[20] John N Tsitsiklis. Efficient algorithms for globally optimal trajectories. IEEE Transactions on Automatic Control, 40(9):1528-1538, 1995.

[21] James A Sethian. A fast marching level set method for monotonically advancing fronts. Proceedings of the National Academy of Sciences, 93(4):1591-1595, 1996.

[22] Hongkai Zhao. A fast sweeping method for eikonal equations. Mathematics of computation, 74(250):603-627, 2005.

[23] Javier V Gómez, David Álvarez, Santiago Garrido, and Luis Moreno. Fast methods for eikonal equations: an experimental survey. IEEE Access, 7:39005-39029, 2019.

[24] Maryam M Najafabadi, Flavio Villanustre, Taghi M Khoshgoftaar, Naeem Seliya, Randall Wald, and Edin Muharemagic. Deep learning applications and challenges in big data analytics. Journal of Big Data, 2(1):1, 2015.

[25] Hyuk Lee and In Seok Kang. Neural algorithm for solving differential equations. Journal of Computational Physics, 91(1):110-131, 1990.

[26] Isaac E Lagaris, Aristidis Likas, and Dimitrios I Fotiadis. Artificial neural networks for solving ordinary and partial differential equations. IEEE transactions on neural networks, 9(5):987-1000, 1998.

[27] Atılım Günes Baydin, Barak A Pearlmutter, Alexey Andreyevich Radul, and Jeffrey Mark Siskind. Automatic differentiation in machine learning: a survey. The Journal of Machine Learning Research, 18(1):5595-5637, 2017.

[28] Julia Ling, Andrew Kurzawski, and Jeremy Templeton. Reynolds averaged turbulence modelling using deep neural networks with embedded invariance. Journal of Fluid Mechanics, 807:155-166, 2016.

[29] Jiequn Han, Arnulf Jentzen, and E Weinan. Solving high-dimensional partial differential equations using deep learning. Proceedings of the National Academy of Sciences, 115(34):8505-8510, 2018.

[30] Justin Sirignano and Konstantinos Spiliopoulos. Dgm: A deep learning algorithm for solving partial differential equations. Journal of Computational Physics, 375:1339-1364, 2018.

[31] Jonathan Tompson, Kristofer Schlachter, Pablo Sprechmann, and Ken Perlin. Accelerating eulerian fluid simulation with convolutional networks. In Proceedings of the 34th International Conference on Machine Learning-Volume 70, pages 3424-3433. JMLR. org, 2017.

[32] Maziar Raissi, Paris Perdikaris, and George E Karniadakis. Physics-informed neural networks: A deep learning framework for solving forward and inverse problems involving nonlinear partial differential equations. Journal of Computational Physics, 378:686-707, 2019.

[33] Jianliang Qian and William W Symes. An adaptive finite-difference method for traveltimes and amplitudes. Geophysics, 67(1):167-176, 2002.

[34] Sergey Fomel, Songting Luo, and Hongkai Zhao. Fast sweeping method for the factored eikonal equation. Journal of Computational Physics, 228(17):6440-6455, 2009. 
[35] Kurt Hornik, Maxwell Stinchcombe, and Halbert White. Multilayer feedforward networks are universal approximators. Neural networks, 2(5):359-366, 1989.

[36] Christopher M Bishop. Pattern recognition and machine learning. Springer, 2006.

[37] P Sibi, S Allwyn Jones, and P Siddarth. Analysis of different activation functions using back propagation neural networks. Journal of Theoretical and Applied Information Technology, 47(3):1264-1268, 2013.

[38] Martín Abadi, Ashish Agarwal, Paul Barham, Eugene Brevdo, Zhifeng Chen, Craig Citro, Greg S. Corrado, Andy Davis, Jeffrey Dean, Matthieu Devin, Sanjay Ghemawat, Ian Goodfellow, Andrew Harp, Geoffrey Irving, Michael Isard, Yangqing Jia, Rafal Jozefowicz, Lukasz Kaiser, Manjunath Kudlur, Josh Levenberg, Dandelion Mané, Rajat Monga, Sherry Moore, Derek Murray, Chris Olah, Mike Schuster, Jonathon Shlens, Benoit Steiner, Ilya Sutskever, Kunal Talwar, Paul Tucker, Vincent Vanhoucke, Vijay Vasudevan, Fernanda Viégas, Oriol Vinyals, Pete Warden, Martin Wattenberg, Martin Wicke, Yuan Yu, and Xiaoqiang Zheng. TensorFlow: Large-scale machine learning on heterogeneous systems, 2015. Software available from tensorflow.org.

[39] George Cybenko. Approximation by superpositions of a sigmoidal function. Mathematics of control, signals and systems, 2(4):303-314, 1989.

[40] Zhou Lu, Hongming Pu, Feicheng Wang, Zhiqiang Hu, and Liwei Wang. The expressive power of neural networks: A view from the width. In Advances in neural information processing systems, pages 6231-6239, 2017.

[41] Charles C Margossian. A review of automatic differentiation and its efficient implementation. Wiley Interdisciplinary Reviews: Data Mining and Knowledge Discovery, 9(4):e1305, 2019.

[42] Adam Paszke, Sam Gross, Soumith Chintala, Gregory Chanan, Edward Yang, Zachary DeVito, Zeming Lin, Alban Desmaison, Luca Antiga, and Adam Lerer. Automatic differentiation in pytorch. In Proceedings of Neural Information Processing Systems, 2017.

[43] David E Rumelhart, Geoffrey E Hinton, and Ronald J Williams. Learning representations by back-propagating errors. nature, 323(6088):533-536, 1986.

[44] Conal Elliott. The simple essence of automatic differentiation. Proceedings of the ACM on Programming Languages, 2(ICFP):1-29, 2018.

[45] Ehsan Haghighat and Ruben Juanes. Sciann: A keras wrapper for scientific computations and physics-informed deep learning using artificial neural networks. arXiv preprint arXiv:2005.08803, 2020.

[46] M Slotnick. Lessons in seismic computing. Soc. Expl. Geophys, 268, 1959.

[47] Cosmin Anitescu, Elena Atroshchenko, Naif Alajlan, and Timon Rabczuk. Artificial neural network methods for the solution of second order boundary value problems. Computers, Materials and Continua, 59(1):345-359, 2019.

[48] Ehsan Haghighat, Ali Can Bekar, Erdogan Madenci, and Ruben Juanes. A nonlocal physics-informed deep learning framework using the peridynamic differential operator. arXiv preprint arXiv:2006.00446, 2020. 